\title{
Vigilância para acidentes e violência: instrumento para estratégias de prevenção e controle
}

\author{
Injury surveillance: \\ a tool for prevention and control strategies
}

Eliseu Alves Waldman 1

Maria Helena de Mello Jorge 1

${ }^{1}$ Departamento de Epidemiologia, Faculdade de Saúde Pública, Universidade de São Paulo, Av. Dr. Arnaldo 715, 01246-904 São Paulo, SP, Brasil. eawaldma@usp.br
Abstract The fast and important increase in morbidity and mortality due to accidents and violence has been one of the most relevant aspects of the epidemiological transition in Brazil since the 80s. This intricate problem calls for social and political measures, and the development of specific legislation and tools for intervening in terms of prevention, treatment, and rehabilitation of those affected, so that risk groups and factors can be identified. The aim of the present paper is to discuss conceptual and operational aspects of using surveillance in adverse health events related to accidents and violence/trauma. Some specific issues related to case definition, identification of sources of information and collecting relevant data are discussed after presenting the concepts, common features and operational aspects related to surveillance systems, regardless of the adverse health events they are aimed at. These issues are discussed in order to try to identify the differences of applying surveillance to accidents and violence in comparison to applying it to infectious diseases. Among the differences one must point out that it is generally necessary to supplement results obtained through surveillance with epidemiological studies in order to identify risk factors.

Key words Surveillance of Injury; Morbidity and Mortality due to Injury; Accidents and Violence
Resumo A rápida e expressiva elevação da morbi-mortalidade por acidentes e violência constituiu um dos pontos mais relevantes da transição epidemiológica no Brasil, a partir dos anos 80. A complexidade do problema implica a aplicação de medidas no âmbito de políticas sociais, elaboração de legislação específica e desenvolvimento de instrumentos de intervenção voltados à prevenção, ao tratamento e à reabilitação dos atingidos, o que pressupõe a identificação de grupos e fatores de risco. O objetivo deste texto é discutir aspectos conceituais e operacionais da utilização da vigilância em eventos adversos à saúde relacionados a acidentes $e$ à violência. Apresentados os conceitos, características comuns e aspectos relativos à operacionalização dos sistemas de vigilância, independente do evento adverso à saúde a que se destinam, foram discutidas algumas questões específicas relativas à elaboração de definições de caso, à identificação de fontes de informação e à coleta dos dados de interesse, buscando identificar diferenças da utilização da vigilância para acidentes e violência se comparadas com a aplicação deste instrumento para doenças infecciosas, salientando entre elas que, para a identificação de fatores de risco, geralmente, se faz necessário complementar os resultados obtidos pela vigilância com pesquisas epidemiológicas. Palavras-chave Vigilância de Causas Externas; Morbi-mortalidade por Causas Externas; Acidentes e Violência 


\section{Introdução}

Um ponto relevante das expressivas modificações dos indicadores de saúde verificadas no Brasil, a partir da década de 80 , foi o acentuado aumento da morbi-mortalidade por causas externas. Tomando a mortalidade no país, no período de 1979 a 1996, é possível verificar que, enquanto no primeiro momento o número de óbitos por acidentes e violências foi de pouco mais de 55 mil, em 1996, atingiu, praticamente, 120 mil. Proporcionalmente ao total de mortes, representaram 8,7\% e em 1996 $13 \%$. Quando calculada sobre o total de óbitos por causas definidas, essa proporção ultrapassou os $15 \%$. Quanto às taxas, o aumento verificado entre os anos estudados, embora não linear, foi de mais de $50 \%$, ao passar de 50 por 100 mil habitantes, no fim da década de 70 , para 76 por 100 mil habitantes, nos últimos anos do período analisado (Mello Jorge \& Gotlieb, 1998).

Comparativamente à região das Américas, as taxas exibidas pelo Brasil somente são comparáveis às da Colômbia (WHO, 1994), salientando-se que, entre 1980 e 1990, só três países americanos apresentaram tendência franca de crescimento: Colômbia, Brasil e Cuba (Yunes \& Rajs, 1994).

Vários estudos têm permitido mostrar que esse comportamento varia segundo o sexo, a idade, a área geográfica e o tipo de causa externa, evidenciando um nítido predomínio de taxas masculinas sobre as femininas e um deslocamento das mortes para faixas etárias mais jovens, comprometendo a esperada elevação da esperança de vida no país, em função da queda acentuada da mortalidade infantil nos últimos vinte anos (Souza, 1994; Mello Jorge, 1998). Entre os tipos de causas externas, têm assumido particular importância os grupos representados pelos acidentes de trânsito e pelos homicídios (em 1996, respectivamente iguais a $29,6 \%$ e $32,7 \%$ ). Estes, especificamente, embora de prevenção muito mais difícil que os primeiros, evidenciam-se como importantes, na medida em que tiveram suas taxas praticamente dobradas nos últimos anos. Essa tendência de crescimento não tem mais se caracterizado somente como um fenômeno urbano, mas vem ocorrendo tanto nas principais capitais do país como em áreas menos desenvolvidas (Mello Jorge et al., 1997).

É sabido, porem, que os dados de mortalidade representam somente a porção mais vi- sível do problema, enquanto a parcela submersa do iceberg continua, em boa parte, desconhecida.

Tomando dados de outros países, onde a morbi-mortalidade por traumas e lesões é expressiva, verifica-se, por exemplo, que nos Estados Unidos, somente em 1986, cerca de 57 milhões de pessoas foram atingidas, com mais de 2 milhões de internações hospitalares. As quedas constituem isoladamente a causa mais freqüente desse grupo de agravos, ou seja, aproximadamente um terço deles (Graitcer, 1992).

No Brasil, somente são conhecidos os dados de hospitalizações pagas pelo Sistema Único de Saúde (SUS), que tem início com o documento Autorização de Internação Hospitalar (AIH), preenchido nos hospitais, obrigatório para a internação de pacientes e para o posterior recebimento de pagamentos referentes a essas internações. Esse sistema engloba cerca de $80 \%$ da assistência médico-hospitalar prestada à população brasileira e representa aproximadamente 13 milhões de internações/ano em mais de 6 mil unidades hospitalares (Levcovitz \& Pereira, 1993).

As internações por lesões e envenenamentos, decorrentes de acidentes e violências, vêm correspondendo a cerca de $6 \%$ do total de internações (693.056 em 1996). A relativamente pequena participação desse grupo de causas, como motivo de internação, é decorrente do fato de as AIH não serem preenchidas para os atendimentos de pronto-socorro, em razão de estes não serem considerados como internações. Este fato subestima, portanto, a real importância da mortalidade por causas externas, visto que é no próprio pronto-socorro que grande número de pacientes tem a resolubilidade de seus casos (Lebrão et al., 1997).

As internações por causas externas revelam que o perfil do paciente é fundamentalmente masculino, com nítida predominância do adulto jovem, sendo de se notar, entretanto, o valor crescente das taxas na população idosa (Lebrão et al., 1997; Mello Jorge \& Gotlieb, 1998)

As fraturas são, em geral, responsáveis por cerca de 38\% das internações, vindo, em seguida as lesões intracranianas e internas e os ferimentos e lesões traumáticas dos vasos, com cerca de 17\% cada (Lebrão et al., 1997).

Considerando-se que as causas externas, em termos de morbi-mortalidade, abrangem desde acidentes de trânsito, acidentes do trabalho até a violência urbana, para citar somente 
as mais freqüentes, não é difícil inferir o amplo espectro de fatores determinantes envolvidos.

No que se refere aos Anos Potenciais de Vida Perdidos (APVP), importante indicador de saúde, é marcante o fato de que, enquanto o país experimentou, entre 1980 e 1991, queda de cerca de $12 \%$ no total de APVP, considerando-se todas as causas de morte, no caso particular dos acidentes e violências o indicador aumentou cerca de 30\% (Iunes, 1997).

Quanto aos custos das lesões e envenenamentos, as limitações dos dados impedem um cálculo mais preciso. Entretanto, seu impacto econômico, medido através dos gastos hospitalares com internações no SUS, mostram que eles se constituem não apenas em importante grupo de causas, mas são também responsáveis por um gasto considerável por parte do governo: 287 milhões de reais em 1994, não incluindo gastos com emergências, despesas com exames e serviços diagnósticos, além de gastos com tratamento ambulatorial e reabilitação. Acresce o fato de que, se por um lado, as internações decorrentes de causas externas tendem a ser mais curtas do que a média dos casos, por outro lado, tendem a consumir recursos de maneira mais intensiva, na medida em que apresentam um gasto/dia aproximadamente $60 \%$ maior do que a média geral paga pelo SUS (Iunes, 1997).

A complexidade do problema, para sua prevenção e controle, implica na aplicação de medidas no âmbito de políticas sociais, na elaboração de legislação específica e no desenvolvimento de instrumentos de intervenção voltados à prevenção, ao tratamento e à reabilitação dos atingidos, o que pressupõe a identificação de grupos e fatores de risco. Por sua relevância, o tema tem despertado a atenção de legisladores, juristas, cientistas sociais e sanitaristas, explicitando a multidisciplinariedade que o assunto envolve. Tais características tornam indispensável a identificação de instrumentos, se possível ágeis, eficientes e de baixo custo, que possam colaborar na fundamentação de estratégias para sua prevenção e controle.

O objetivo deste texto é discutir aspectos conceituais e operacionais da utilização da vigilância em eventos adversos à saúde relacionados a acidentes e violência, apresentando vantagens e limitações desse instrumento.

\section{Características gerais da vigilância como instrumento de saúde pública}

\section{Conceitos}

Existem dois conceitos de vigilância consagrados em saúde pública. O "clássico", que surgiu no final do século passado, com a ampliação do conhecimento a respeito das doenças infecciosas, fundamentado no desenvolvimento da microbiologia, pode ser definido pela específica mas limitada função de observar contatos de pacientes atingidos pelas chamadas doenças pestilenciais como a cólera, a varíola e a peste. Seu propósito era detectar os primeiros sintomas para a rápida instituição do isolamento (Langmuir, 1976).

O outro conceito, chamado "moderno", surgiu no início da década de 50, tendo sido definido por Alexander Langmuir (1963), como a observação contínua da distribuição e tendências da incidência de eventos adversos à saúde mediante a coleta sistemática, consolidação e avaliação de informes de morbidade e mortalidade, assim como de outros dados relevantes $e$ a regular disseminação dessas informações analisadas a todos que necessitam conhecê-la”. Portanto, a vigilância deixa de se preocupar com contatos de doenças infecciosas e passa a acompanhar o comportamento de eventos específicos adversos à saúde na comunidade, com vistas a recomendar, em tempo oportuno, estratégias para sua prevenção e controle, fundamentadas no conhecimento científico.

$\mathrm{Na}$ década de 60 , a vigilância, neste novo conceito, recebe o qualificativo epidemiológica (Raska, 1964), consagrado internacionalmente pela sua ampla utilização durante a campanha de erradicação da varíola. Mais recentemente, Thacker e Berkelman (1988), abordando uma antiga discussão a respeito da adequação deste qualificativo, afirmaram que o uso do termo epidemiológica, para qualificar vigilância, é equivocado, uma vez que epidemiologia é uma disciplina abrangente, que incorpora a pesquisa e cuja aplicação nos serviços de saúde vai além do instrumento de saúde pública denominado vigilância. Argumentaram ainda que a utilização desse qualificativo tem induzido freqüentemente a confusões que restringem a aplicação da epidemiologia nos serviços ao acompanhamento de eventos adversos à saúde, atividade que constitui somente parte das aplicações da epidemiologia em saúde pública. 
Fundamentados nesta argumentação, Thacker e Berkelman (1988) propuseram a adoção da denominação de vigilância em saúde pública para essa particular aplicação da epidemilogia em serviços de saúde, como forma de evitar confusões a respeito da sua precisa delimitação. Como conseqüência, ficou consagrada internacionalmente a denominação vigilância em saúde pública, utilizada em praticamente todas as publicações sobre o assunto, desde o início dos anos 90, inclusive em documento recente da Organização Pan-Americana de Saúde (OPAS) (Teutsch \& Thacker, 1995). Vale dizer que essa alteração da denominação não implicou na adoção de uma nova abordagem ou em modificações de aspectos conceituais ou operacionais da vigilância.

Utilizando o enfoque sistêmico e sintetizando as diversas experiências e concepções da vigilância, no seu conceito "moderno", desenvolvidas nos últimos 40 anos, em diferentes partes do mundo, e preocupados com uma concepção adequada, em suas linhas gerais, a um sistema de saúde descentralizado e com um desempenho que lhe confira um mínimo de auto-sustentação técnica, pode-se dizer que a vigilância de um específico evento adverso à saúde é composta ao menos por dois subsistemas (Waldman, 1998).

Subsistema de informações para a agilização das ações de controle: situa-se nos níveis locais dos serviços de saúde e tem por objetivo agilizar o processo de identificação e controle de eventos adversos à saúde. A equipe que faz parte desse subsistema deve estar perfeitamente articulada com a de planejamento e avaliação dos programas, devendo, portanto, assumir parte das responsabilidades pela elaboração das normas utilizadas no nível local dos serviços de saúde.

Subsistema de inteligência epidemiológica: é especializado e tem por função elaborar as bases técnicas dos programas de controle de específicos eventos adversos à saúde a partir da análise sistemática do seu comportamento na comunidade, fundamentada no conhecimento científico e tecnólogico disponível.

Outro objetivo do subsistema de inteligência epidemiológica é identificar "lacunas" no conhecimento científico e tecnológico, à medida que detecte mudanças desse comportamento não explicadas pelo conhecimento científico disponível. Feito isso, é papel da inteligência epidemiológica induzir a pesquisa.
Esse subsistema exerce também a função de incorporar aos serviços de saúde o novo conhecimento produzido pela pesquisa, com o objetivo de aprimorar continuamente as medidas de controle. Isso pode ser feito, incluindo-o nas recomendações técnicas que são encaminhadas aos serviços de saúde na forma de boletins epidemiológicos. Constitui-se, também, a ponte entre o subsistema de serviços de saúde e o subsistema de pesquisa de um sistema nacional de saúde, uma vez que pode assumir o papel de indutor de linhas de pesquisa que respondam a questões prioritárias ou de importância emergente em saúde pública e, por outro lado, incorpora o conhecimento produzido ao atualizar continuamente as bases técnicas dos programas de controle de doenças (Waldman, 1998).

\section{Principais características}

Como instrumento, a vigilância em saúde pública, independente do particular evento adverso à saúde a que se aplica, apresenta algumas características comuns: a) deve ser simples; b) contínua (aspecto que a diferencia da pesquisa); c) deve ter obrigatoriamente três componentes: a informação, a análise regular da informação com fundamento no conhecimento científico e a ampla disseminação da informação analisada a todos que dela necessitam.

A vigilância não se restringe a um sistema de informação para agilização das ações de controle, pois deve, além disso, constituir o instrumento que oferece as bases técnicas dos programas de controle de específicos agravos. É um instrumento ágil e de baixo custo aplicado ao contínuo aperfeiçoamento técnico dos serviços de saúde (Foege et al., 1976; Thacker \& Berkelman, 1988).

\section{Operacionalização}

A operacionalização de um sistema de vigilância para um específico evento adverso à saúde implica na avaliação da relevância em saúde pública do evento para o qual se pretende implantar o sistema, na clara delimitação de seus objetivos e na definição de caso perfeitamente adequado aos objetivos do sistema de vigilância proposto. Estabelecidos a importância, os objetivos e a definição de caso, deve-se passar a identificar os componentes do sistema: 1) a população alvo desse sistema de 
vigilância; 2) a periodicidade da coleta de informações; 3 ) que informações serão coletadas; 4) qual é a fonte dessas informações; quem provê a informação para o sistema; 5) como a informação será coletada; 6) como é transferida a informação; 7) quem analisa as informações; 8) como são analisadas as informações; 9) com que freqüência são analisadas as informações; 10) com que freqüência são difundidos os relatórios; 11) qual o instrumento a ser utilizado para ampla disseminação da informação analisada (CDC, 1988).

Outro aspecto a ser definido é o tipo de vigilância, ou seja, a opção será por um sistema passivo ou ativo. Resumidamente, o que caracteriza a vigilância passiva é a notificação espontânea, enquanto na vigilância ativa temse o contato regular entre a equipe da vigilância e as equipes das fontes de informação (Teutsch \& Churchil, 1994).

Para o delineamento adequado de um sistema de vigilância, além dos pontos já assinalados, é fundamental analisar, já na fase de planejamento, as possíveis repercussões de cada opção efetuada, levando em conta os indicadores utilizados na avaliação da vigilância. Sem entrar em detalhes, mas remetendo para bibliografia específica (CDC, 1988; Waldman \& Rosa, 1998), devem ser citados os seguintes indicadores: utilidade, oportunidade, aceitabilidade, simplicidade, flexibilidade, representatividade, sensibilidade, e valor preditivo positivo.

Sumariando as questões relativas à avaliação de sistemas de vigilância, cabe salientar que os diversos atributos apresentados são interdependentes, uma vez que o aprimoramento de um pode comprometer o outro. Quando se aumenta a sensibilidade de um sistema para detectar uma grande proporção de casos de um determinado evento adverso à saúde, geralmente, se está aprimorando a representatividade e a utilidade do sistema. Porém, nesse caso, tem-se um aumento do custo e um aumento dos casos falso-positivos. Portanto, é importante não analisar individualmente o desempenho alcançado por esses atributos, mas sim buscar um equilíbrio apropriado entre eles, tomando em consideração o objetivo do sistema de vigilância.

Para demonstrar a importância dessa análise preliminar, já na fase do esboço do sistema, podem ser apresentados alguns exemplos. Se a opção for por uma definição de caso muito específica, o sistema provavelmente apre- sentará um valor preditivo positivo elevado, mas uma sensibilidade baixa. Por outro lado, os sistemas passivos costumam ser simples, de baixo custo, mas apresentam mau desempenho quanto à sensibilidade e representatividade. Já os sistemas ativos apresentam um custo mais elevado, mas são mais sensíveis e representativos.

Embora os sistemas de vigilância não precisem ser perfeitos para serem úteis, muitas vezes certas limitações impedem que esse instrumento tenha a utilidade necessária que justifique sua implementação.

Os fatores que mais freqüentemente levam a limitações do desempenho de sistemas de vigilância são a subnotificação; a baixa representatividade; o baixo grau de oportunidade; a inconsistência da definição de caso (CDC, 1992).

A subnotificação geralmente decorre do fato de os sistemas de vigilância, em sua maioria, serem passivos. É freqüente nesses casos ela atingir níveis superiores a $50 \%$ ou $70 \%$ dos casos, determinando o retardo ou mesmo a ausência de ações de controle.

A subnotificação está freqüentemente relacionada com a) a falta de conhecimento, por parte dos profissionais de saúde, da importância e dos procedimentos necessários para a notificação; b) o desconhecimento da lista de doenças submetidas à vigilância; c) a ausência de adesão à notificação, pelo tempo consumido no preenchimento da ficha e pela ausência do retorno da informação analisada com as recomendações técnicas pertinentes; d) a preocupação dos profissionais de saúde com a quebra da confidencialidade das informações; e) falta de percepção dos profissionais, da relevância em saúde pública, das doenças submetidas à vigilância.

A baixa representatividade de um sistema de vigilância pode resultar da falta de homogeneidade da subnotificação, dificultando a identificação de tendências, grupos e fatores de risco.

Os dois erros sistemáticos mais observados são: 1) a tendência a notificar mais os casos de maior gravidade e os hospitalizados do que os de características benignas; 2) notifica-se com maior intensidade doenças que estão sendo focalizadas pelos meios de comunicação.

A falta de oportunidade de um sistema de vigilância pode ocorrer em diferentes momentos por diversos motivos: a) dificuldade, em alguns casos, de se obter o diagnóstico antes da confirmação laboratorial; b) ineficiência 
dos serviços no procedimento de notificação; c) demora na análise, problema freqüente quando o sistema de vigilância é uma atividade mais burocrática que técnica voltada ao apoio dos serviços de saúde; d) o retardo em qualquer fase do sistema que leva a uma demora na disseminação da informação analisada, impedindo que a população e os profissionais de saúde tenham as informações indispensáveis para uma ação oportuna e eficiente.

A inconsistência da definição de caso leva a vigilância a confirmar os casos aceitando o diagnóstico dos clínicos, independentemente da forma como eles foram efetuados. Como exemplo da necessidade de uma padronização dessa definição, podem ser citados os critérios de confirmação das meningites assépticas que podem variar de local para local e de um médico para outro.

\section{Vigilância de acidentes e violência}

\section{Aspectos específicos}

A vigilância de eventos específicos adversos à saúde relacionados a acidentes e violência deve ter por objetivo oferecer às autoridades sanitárias os seguintes subsídios para a elaboração de programas de prevenção e controle: estimar a morbi-mortalidade em termos de magnitude, tendências, custo e impacto social; identificar grupos e fatores de risco; detectar clusters; avaliar a efetividade de intervenções; induzir pesquisas e incorporar novos conhecimentos visando ao contínuo aperfeiçoamento dos serviços de saúde. Além disso, a vigilância deve contribuir para o aprimoramento dos cuidados médicos na fase aguda, no tratamento e na reabilitação dos atingidos (Graitcer, 1992).

A utilização da vigilância para eventos adversos relacionados a acidentes e violência é relativamente recente e apresenta algumas peculiaridades se comparada com a sua aplicação em doenças infecciosas.

\section{Definição de caso}

De acordo com o assinalado no início, temse um amplo espectro de agravos rotulados genericamente como causas externas. Quando se necessita definir caso, para um particular sistema de vigilância, deve-se decidir em função do seu objetivo. Dado que o aspecto prin- cipal de interesse para a vigilância é o epidemiológico, a definição de caso deverá relacionar-se ao fato ou fator que induz ao acidente ou violência. Exemplo: as quedas ou acidentes por veículo a motor. Nesse ponto, existem significativas diferenças se comparadas com a vigilância para doenças infecciosas, onde a definição de caso abrange sinais, sintomas clínicos e dados laboratoriais.

Conforme os objetivos do sistema, a definição de caso necessita ser mais delimitada, especificando, por exemplo, o nível da assistência médica em que os casos são atendidos: se hospital, pronto-socorro, consultório médico, etc.; a evolução do caso: recuperação completa, paralisia temporária ou permanente, óbito; a relação com algum tipo de atividade: esporte, lazer, trabalho; características específicas do evento: queda da escada ou queda de uma motocicleta; freqüentemente são levados em conta os fatores de risco: abuso de álcool ou drogas, e os fatores de proteção: uso de cinto de proteção ou detetor de fumaça. Se o objetivo do sistema for determinar a ocorrência do evento na comunidade, a definição do caso deverá abranger todos os casos independente da gravidade (Graitcer, 1992).

Ainda com referência à definição de caso para sistemas de vigilância para acidentes e violência, com o intuito de padronizar conceitos, é recomendável adotar aquele proposto pela Organização Mundial da Saúde (OMS) que inclui neste grupo de eventos adversos à saúde todo tipo de agravo não natural, ou seja causas externas de lesões e envenenamentos (OMS, 1995).

\section{Fontes de dados}

Os sistemas de vigilância para acidentes e violência utilizam freqüentemente como fontes de dados atestados de óbito, prontuários médicos e hospitalares e inquéritos domiciliares. Em virtude dos dados utilizados por sistemas de vigilância de acidentes e violência terem sido colhidos com outros objetivos que não a identificação de fatores de risco e a prevenção desses eventos, geralmente eles são falhos por serem incompletos, imprecisos, às vezes desatualizados, o que constitui fator limitante para os objetivos do sistema de vigilância em questão (Graitcer, 1987). Com o objetivo de diminuir essas limitações, têm sido desenvolvidas algumas iniciativas de introdução de modificações no conjunto de variáveis con- 
tidas nesses bancos de dados de forma a torná-los mais adequados aos propósitos de diferentes sistemas de vigilância para acidentes e violência, visando a interligação de bancos de dados que sejam complementares considerando o interesse da vigilância (Thacker \& Berkelman, 1988).

As principais fontes de informação para o conhecimento de eventos adversos associados à acidentes e violência sobre as pessoas são:

Sistema de Informações Hospitalares do Sistema Único de Saúde (SIH/SUS) - É o já referido sistema dos hospitais próprios ou conveniados com o SUS que, embora tenha fundamentalmente uma lógica voltada para a área administrativa, tem representado excelente fonte de informações dos casos internados. Como limitação, apresenta o fato de só se referir aos hospitais do SUS, além da incoveniência de não abranger dados de pronto-socorro.

Sistema de Informação de Mortalidade do Ministério da Saúde (SIM/MS) - Está implantado no país desde 1975 e é gerido pelo Centro Nacional de Epidemiologia. Abrange cerca de 900 mil óbitos/ano. Entretanto, ainda está incompleto para algumas áreas das Regiões Norte e Nordeste. Relativamente às causas externas, a fonte é representada pelos institutos de medicina legal. Em cerca de 10\% dos casos média para o país - as causas das mortes não estão detalhadas, distorção que vem sendo minimizada com o auxílio de informações adicionais.

Comunicação de Acidentes do Trabalho/ CAT - Trata-se de um sistema de abrangência nacional, gerido pelo Instituto Nacional de Seguro Social/INSS. Sua maior limitação diz respeito ao fato de essa fonte excluir os trabalhadores autônomos, aqueles sem carteira assinada e os vinculados a outros sistemas previdenciários.

Boletim do Departamento Nacional de Estradas de Rodagem (DNER) - O DNER coleta e analisa dados relativos a acidentes de trânsito ocorridos em rodovias federais e completa seus dados com informações análogas procedentes dos níveis estadual e municipais.

Boletim de Ocorrência Policial (BO) - É o instrumento usado nas delegacias de polícia, em nível estadual/municipal, não havendo, para o mesmo, qualquer tipo de padronização.

Sistema Nacional de Informações TóxicoFarmacológica (SINITOX) - Existe desde 1980 e foi criado para ter abrangência nacional. Divulga casos e óbitos por intoxicação humana no país, embora só contasse, até 1997, com 31 centros de controle de intoxicação distribuídos em 16 estados da federação.

\section{Coleta de dados}

Embora a escolha das variáveis para um específico sistema de vigilância para acidentes e lesões dependa dos objetivos deste e das características do evento objeto da vigilância, é fundamental a obtenção de um conjunto mínimo de dados indispensáveis para uma análise epidemiológica, ou seja, que forneça informações básicas relativas à pessoa, ao tempo, à natureza e à causa imediata do trauma ou lesão, do diagnóstico, das conseqüências imediatas, de médio e longo prazo derivadas do evento (tipo de tratamento, hospitalização, perda temporária ou permanente de função, óbito, etc.), a fatores ou condições no momento do evento (uso de cinto de segurança, uso de capacete no caso de motociclistas, presença de efeitos de álcool ou de drogas).

Com relação à coleta de dados algumas considerações são necessárias para esclarecer problemas decorrentes das diferentes lógicas da OMS ao estabelecer regras quanto à codificação de acidentes e violências em mortalidade e em morbidade.

Para o primeiro caso é válido o conceito de causa básica de morte, definido como a) " $a$ doença ou lesão que iniciou a cadeia de acontecimentos patológicos que conduziram diretamente à morte” ou b) "as circunstâncias do acidente ou violência que produziram a lesão fatal” (OMS, 1995). É importante salientar que do ponto de vista da prevenção da morte, é necessário interromper a cadeia de eventos mórbidos ou instituir a cura em algum ponto e aqui se entende que o objetivo mais eficaz em saúde pública é prevenir a ação da causa precipitante. Assim, quando a causa a ser codificada como básica for uma lesão ou outro efeito de uma causa externa classificada no capítulo XIX (Lesões, Envenenamento e Algumas Outras Conseqüências de Causas Externas $\mathrm{S}, \mathrm{T}$ ), as circunstâncias que deram origem a essa afecção deverão ser selecionadas como causa básica para tabulação e codificadas no capítulo XX (Causas Externas de Morbidade e de Mortalidade, V, W, Z e Y), ambos da Classificação Estatística Internacional de Doenças e Problemas Relacionados à Saúde, Décima Revisão (OMS, 1995), podendo o código para a lesão ou efeito ser usado como adicional. 
Com relação à morbidade, o que deve ser codificado diz respeito à afecção principal tratada ou investigada durante um episódio relevante de atenção à saúde; no caso específico de causas externas, a lesão primariamente responsável pela necessidade de tratamento ou investigação.

Isso quer significar que se, por exemplo, um paciente for internado com uma ruptura de fígado decorrente de um acidente de trânsito e vier a óbito, do ponto de vista da morbidade, o diagnóstico principal será a ruptura de fígado e a causa básica a ser selecionada na declaração de óbito diz respeito ao acidente de trânsito.

Esse raciocínio dicotômico, até bem recentemente, estava impossibilitando o estudo conjunto da morbi-mortalidade por essas causas, com visível prejuízo, inclusive no sentido de conhecer as lesões mais freqüentes, tempo de permanência em hospital, procedimentos e gastos para cada um dos tipos de acidentes/ violência. Entretanto, a partir de 1998, em razão de Portaria do Ministério da Saúde (Portaria 142 de 13 de novembro de 1997), os hospitais passaram a ter que registrar tanto a natureza da lesão como as circunstâncias que originaram essa condição (no caso específico do exemplo, a ruptura de fígado e o acidente de trânsito).

\section{Etiologia}

A rede da causalidade de acidentes e violências é complexa. Portanto, para descrever a complicada interação de fatores de risco envolvidos na ocorrência de traumas e lesões com o objetivo de estabelecer medidas adequadas de intervenção, pode ser necessário complementar os dados gerados por sistemas de vigilância com informações obtidas de minuciosos estudos epidemiológicos, como por exemplo estudos tipo caso-controle (Graitcer, 1992).

\section{Considerações finais}

O crescimento dos acidentes e violências entre nós e a relativa inconsistência de dados referentes a esses eventos somados à ausência de alguns registros e o conhecimento sobre certos agravos, principalmente no que diz respeito à determinação de fatores de risco associados a cada um deles, têm desencadeado algumas tentativas pontuais de vigilância desses agravos.
É o caso, por exemplo, da Secretaria $\mathrm{Mu}$ nicipal de Recife que, associada ao Centro Latino-Americano de Violência e Saúde Jorge Careli (Claves), da Fundação Oswaldo Cruz, promoveu o Curso de Vigilância em Violência e Saúde, de outubro a dezembro de 1997, na cidade de Recife e que teve como principal objetivo a formação de seus profissionais e a sensibilização dos envolvidos para o tema da violência, bem como a montagem do monitoramento dos dados.

Em São Paulo, a Secretaria de Estado da Saúde "estabeleceu a necessidade de desenvolver um projeto de vigilância para os homicídios, com a finalidade de acompanhamento ágil das informações, possibilitando um conhecimento fidedigno do problema para a identificação dos principais fatores de risco e a proposição de ações que visem ao seu controle". Tal projeto, que envolveu técnicos das secretarias de Saúde, Justiça e Cidadania e segurança pública, em parceria com a Universidade de São Paulo, Faculdade de Medicina da Santa Casa e com o Programa de Aprimoramento das Informações de Mortalidade da Prefeitura do Município de São Paulo, partiu de uma proposta de vigilância da OPAS e elaborou um software ligando as informações provindas de diferentes fontes.

O pré-teste foi realizado com 1.490 mortes por homicídios ocorridas nos meses de julho, agosto e setembro de 1997, no município. O conhecimento de como as instituições coletam e trabalham as informações permitiu reelaborar o instrumento inicial, rediscutir as variáveis anteriormente estabelecidas e tentar uma unificação das estatísticas, com vistas ao projeto definitivo (Gawryzewski, 1998).

Diante da necessidade de elaborar estratégias bem fundamentadas para o desenvolvimento de programas de prevenção e controle de eventos adversos à saúde determinados por causas externas, é recomendável a utilização regular da vigilância com essa finalidade, em face da sua simplicidade e baixo custo, ainda que sejam conhecidas suas limitações, o que torna freqüentemente indispensável a complementação de seus resultados com o desenvolvimento de pesquisas epidemiológicas. 


\section{Referências}

CDC - Centers for Disease Control and Prevention 1988. Guidelines for evaluating surveillance systems. Mor bidity and Mortality Weekly Report 37(5S): 1-18.

CDC - Centers for Disease Control and Prevention 1992. Principles of Epidemiology. An Introduction to Applied Epidemiology and Biostatistics, p. 289-345. Ed. Public Health Service/CDC, 2nd ed., Atlanta.

Foege WH, Hogan RC \& Newton LH 1976. Surveillance projects for selected diseases. International Journal of Epidemiology 5: 29-37.

Graitcer PL 1987. Development of state and local injury surveillance system. Journal of Safety Research 18: 191-198.

Graitcer PL 1992. Injury surveillance, p. 142-156. In W Halperin \& EL Baker Jr Public Health Surveillance. Van Nostrand Reinold, New York.

Gawryszewski VP 1998. Uma proposta para a vigilância epidemiológica dos homicídios em São Paulo, p. 80. IV Congresso Brasileiro de Epidemiologia, V. 1, Rio de Janeiro (CC 55-185).

Iunes RF 1997. Impacto econômico das causas externas no Brasil: esforço de mensuração. In MHP Mello Jorge \& R Laurenti, Acidentes e violência no Brasil. Revista de Saúde Pública 31(Supl. 4): 38-46.

Langmuir AD 1963. The surveillance of communicable diseases of national importances. New England Journal of Medicine 268(4): 182-192.

Langmuir AD 1976. Willian Farr: founder of modern concepts of surveillance. International Journal Epidemiology 5: 13-18.

Lebrão ML, Mello Jorge MHP \& Laurenti R 1997. Morbidade hospitalar por lesões e envenenamentos. In MHP Mello Jorge \& R Laurenti, Acidentes e violência no Brasil. Revista de Saúde Pública 31(Supl. 4): $26-37$

Levcovitz E \& Pereira TRC 1993. SIH/SUS (Sistema AIH): uma Análise do Sistema Público de Remuneração de Internações Hospitalares no Brasil, 19831991. Universidade Estadual do Rio de Janeiro/Instituto de Medicina Social.

Mello Jorge MHP 1998. Como morrem os nossos jovens. In Jovens Acontecendo na Trilha das Politica Públi cas. Comissão Nacional de População e Desenvolvimento, Brasília.
Mello Jorge MHP \& Gotlieb SLD 1998. As Condições de Saúde no Brasil, São Paulo. Relatório final - Projeto financiado pelo CNPq, processo No. 520311/96-5.

Mello Jorge MHP, Gawryszewski VP \& Latorre MRDO. 1997. Análise dos dados de mortalidade. In MHP Mello Jorge \& R Laurenti, Acidentes e violência no Brasil. Revista de SaúdePública 31(Supl. 4): 5-25.

OMS - Organização Mundial de Saúde 1995. Classificação Estatística Internacional de Doenças e Problemas Relacioandos à Saúde - 10a. Revisão. Centro Colaborador da Organização Mundial da Saúde para Classificação de Doenças em Português, São Paulo.

Raska K 1964. The epidemiological surveillance programme. Journal of Hygiene and Epidemiology, Praha, $8:$ 137-168.

Souza ER 1994. Homicídio: o grande vilão da saúde pública na década de 80. Cadernos de Saúde Pública 10(Supl.1): 135-45

Teutsch SM \& Churchil RE 1994. Principles and Practice of Public Health Surveillance. Oxford University Press, New York, 274 pp.

Teutsch SM \& Thacker SB 1995. Planificación de un sistema de vigilancia en salud pública. Boletín Epidemiológico. Organización Panamericana de la Salud 16(1): 1-7.

Thacker SB \& Berkelman RL 1988. Public health surveillance in the United States. Epidemiology Reviews 10:164-190.

Waldman EA 1998. Usos da vigilância e da monitorização em saúde pública. Informe Epidemiológico do SUS 5(3): 87-107.

Waldman EA \& Rosa TEC 1998. Vigilância em Saúde Pública. Fundação Peirópolis, São Paulo.

WHO - World Health Organization 1994. World Health Statistics Annual, 1993, Geneve.

Yunes J \& Rajs D 1994. Tendência de la mortalidad por causas violentas en la populación general y entre los adolescentes y jovenes de las Americas. Cadernos de Saúde Pública 10(Supl. 1): 88-125. 